\title{
Correlation between Sestrin-2 and PERK Signaling in Matured Porcine Oocytes according to ER-stress during In Vitro Maturation
}

\author{
Hyo-Jin Park ${ }^{1,2, \#}$, In-Su Kim ${ }^{1,2, \#}$, Jin-Woo Kim ${ }^{1,2}$, Seul-Gi Yang, ${ }^{1,2}$, Min-Ji Kim ${ }^{1,2}$ and \\ Deog-Bon $\mathrm{Koo}^{1,2, *}$ \\ ${ }^{1}$ Department of Biotechnology, College of Engineering, Daegu University, Gyeongsan 38453, Korea \\ ${ }^{2}$ Institute of Infertility, Daegu University, Gyeongsan 38453, Korea
}

Received September 11, 2019

Revised September 23, 2019

Accepted September 24, 2019

\section{*Correspondence \\ Deog-Bon Koo \\ E-mail:dbkoo@daegu.ac.kr}

\section{ORCID}

https://orcid.org/0000-0001-7825-9598

\#The first two authors contributed equally to this work.

\begin{abstract}
Sestrin-2 (SESN2) as a stress-metabolic protein is known for its antioxidative effects as a downstream factor of PERK pathways in mammalian cells. However, the expression patterns of SESN2 in conjunction with the UPR signaling against to ER stress on porcine oocyte maturation in vitro, have not been reported. Therefore, we confirmed the expression pattern of SESN2 protein, for which to examine the relationship between PERK signaling and SESN2 in porcine oocyte during IVM. We investigated the SESN2 expression patterns using Western blot analysis in denuded oocytes (DOs), cumulus cells (CCs), and cumulus-oocyte complexes (COCs) at 22 and $44 \mathrm{~h}$ of IVM. As expected, the SESN2 protein level significantly increased ( $p<$ 0.01 ) in porcine COCs during $44 \mathrm{~h}$ of IVM. We investigated the meiotic maturation after applying ER stress inhibitor in various concentration (50, 100 and $200 \mu \mathrm{M})$ of tauroursodeoxycholic acid (TUDCA). We confirmed significant increase $(p<0.05)$ of meiotic maturation rate in TUDCA $200 \mu \mathrm{M}$ treated COCs for $44 \mathrm{~h}$ of IVM. Finally, we confirmed the protein level of SESN2 and meiotic maturation via regulating ER-stress by only tunicamycin (Tm), only TUDCA, and Tm + TUDCA treatment in porcine COCs. As a result, treatment of the TUDCA following Tm pre-treatment reduced SESN2 protein level in porcine COCs. In addition, SESN2 protein level significantly reduced in only TUDCA treated porcine COCs. Our results suggest that the SESN2 expression is related to the stress mediator response to ER stress through the PERK signaling pathways in porcine oocyte maturation.
\end{abstract}

Keywords: ER-stress, in vitro maturation, PERK signaling, porcine oocyte, Sestrin-2

\section{INTRODUCTION}

Accumulation of unfolded proteins in endoplasmic reticulum (ER) lumen causes ER stress (Kaufman, 1999). Under ER stress condition, unfolded protein response (UPR), a cellular protective response, is activated through three transmembrane stress sensors such as a protein $\mathrm{ki}^{-}$ nase RNA-like ER kinase (PERK), activating transcription factor-6 (ATF6), and inositol-requiring protein 1 (IRE1) signaling pathways (Rasheva and Domingos, 2009; Walter and Ron, 2011). Above all, PERK sensors initiate protective response including attenuation of protein translation, increasing molecular chaperone genes expression levels (Bravo et al., 2013). In addition, the PERK signal controls 
Park et al. SESN2 and PERK Signaling in Matured Porcine Oocyte

cell-cycle, chaperon proteins activity, and anti-oxidant enzymes for reducing ER stress in mammalian cells (Brewer and Diehl, 2000).

These UPR signaling activity play important roles for oocyte maturation, cumulus cells expansion, and early embryonic development in female reproduction system (Yang et al., 2016). Many previous studies demonstrated that the three UPR signal factors activations are linked closely to oocyte quality, meiotic maturation, and blastocyst development of implantation embryos in pigs (Luo et al., 2006; Lin et al., 2015). Our previous study showed that the regulation of UPR signaling against to ER stress improves the meiotic maturation and cumulus cell expansion in porcine COCs during in vitro maturation (IVM) progression (Park et al., 2018).

Sestrin-2 (SESN2) as an antioxidant protein is an essential that is induced by oxidative stress and protects response to reactive oxygen species (ROS) (Kim et al., 2015). In mammalian cells, the SESN2 is known as the stress inducible proteins for regulating cellular metabolic homeostasis and nuclear factor-E2-related factor 2 (Nrf2)-related antioxidant signaling (Shin et al., 2012). Under the oxidative stress and severe ROS induced ER stress, SESN2 as a cellular stress regulator performed antioxidative roles through PERK signaling pathway in human colorectal cancer cells (Jin et al., 2019). Recently, Ye et al. reported that SESN2 induced the chaperone function and anti-oxidative enzymes expression through downstream of PERK/eIF-2/ATF4 signaling against ER stress (Ye et al., 2015). Reduction of SESN2 gene expression using siRNA increased ER stress-mediated apoptosis (Kim et al., 2014). However, the effects of SESN2 on oocytes maturation via UPR signaling and ER stress during IVM of porcine oocyte have not been reported.

In present study, we first investigated the changes of SESN2 expression pattern in matured porcine COCs during IVM progression. Also, we confirmed whether the conditions of ER stress regulators (such as ER stress inducer, tunicamycin: Tm, and ER stress inhibitor, tauroursodeoxycholic acid: TUDCA) regulate PERK/eIF-2/ATF4 signaling pathways and SESN2 expression. In addition, we investigated that regulation PERK pathways by ER stress leads to SESN2 expression induction in porcine COCs after $44 \mathrm{~h}$ of IVM.

\section{MATERIALS AND METHODS}

\section{Chemicals}

Unless otherwise stated, all chemicals and reagents used were purchased from Sigma-Aldrich (St. Louis, MO, USA).

\section{In vitro maturation (IVM)}

Porcine ovaries were collected from local slaughterhouses (Gyeongsan, Gyeongbuk, Korea) and transported to the laboratory in a thermos containing $0.9 \%$ saline supplemented with $75 \mu \mathrm{g} / \mathrm{mL}$ potassium penicillin $\mathrm{G}$ at approximately $30-35^{\circ} \mathrm{C}$. Transported ovaries were washed three times with saline and follicular fluid was collected from follicles that were 3 to $6 \mathrm{~mm}$ in diameter using an 18 -gauge needle attached to a $10 \mathrm{~mL}$ syringe. The collected follicular fluid had a 15 min settling time and then the supernatant was removed. The remaining pellet was diluted in Tyrode's lactate-N-2-hydroxyethylpiperazineethanesulfonic acid (TL-HEPES), and immature cumulusoocyte complexes (COCs) were selected using an optical microscope as previously described (Park et al., 2018). Approximately 50 to 60 immature COCs were cultured in 500 $\mu \mathrm{L}$ of IVM medium in a four-well multi-dish at $38.5^{\circ} \mathrm{C}$ in an atmosphere of $5 \% \mathrm{CO}_{2}$ after three washes in TL-HEPES and IVM medium. During the $22-\mathrm{h}$ maturation, North Carolina State University-23 (NCSU-23) medium supplemented with $10 \%(\mathrm{v} / \mathrm{v})$ porcine follicular fluid, $10 \mathrm{ng} / \mathrm{mL}$ epidermal growth factor (EGF), $25 \mu \mathrm{M} \beta$-mercaptoethanol, $10 \mathrm{IU} / \mathrm{mL}$ human chorionic gonadotropin (hCG), $10 \mathrm{IU} /$ $\mathrm{mL}$ pregnant mare's serum gonadotropin (PMSG), and $0.57 \mathrm{mM}$ cysteine was used for oocyte maturation. After maturation, COCs were additionally cultured in PMSG and hCG-free maturation medium for $22 \mathrm{~h}$. During the maturation periods, $\mathrm{Tm}(1$ and $5 \mu \mathrm{g} / \mathrm{mL})$ and/or TUDCA (50, 100 and $200 \mu \mathrm{M})$ as an ER stress regulators were added to the maturation medium according to the treatment timing during three period of IVM such as IVM I (0-22 h), IVM II (22-44 h) and IVM I \& IVM II (0-44 h) (Fig. 1). In present study, we used to the treatment concentration of TUDCA treatment concentration as descripted by Kim et al., 2012 (Kim et al., 2012).

\section{Protein extraction and Western blot analysis}

Matured COCs (25 per group) lysates were extracted using protein extraction solution and PRO-PREP protein lysis buffer (iNtRON, Daejeon, Korea). The sample lysates 


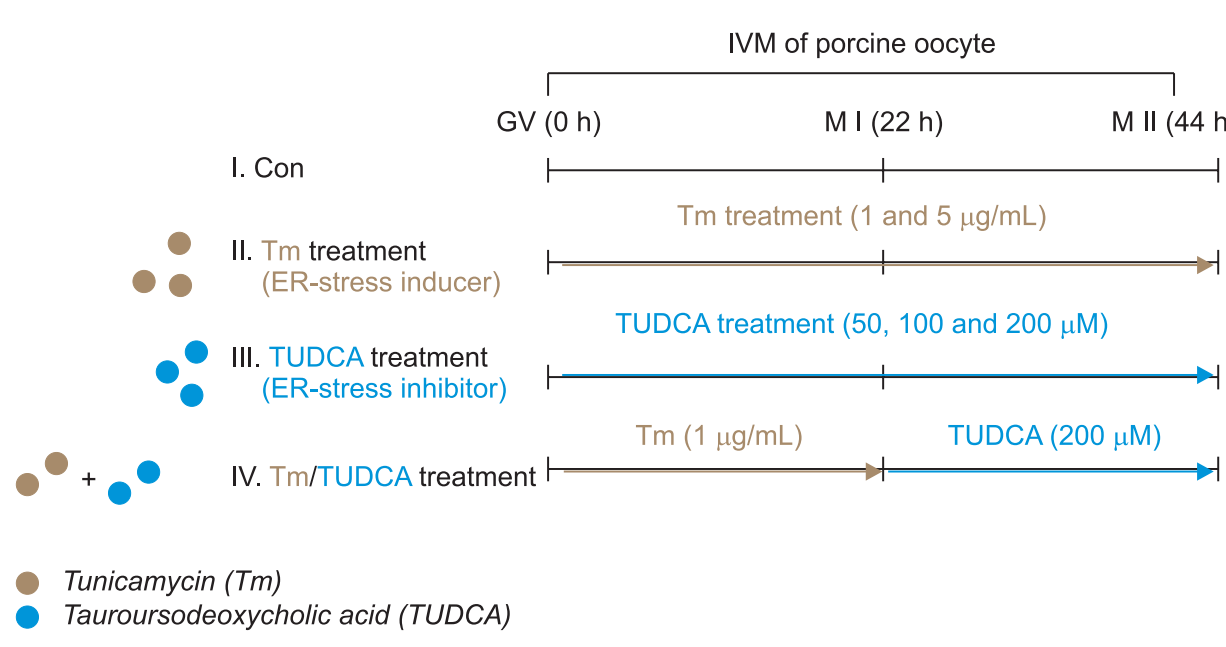

Fig. 1. Graphical description of Tm and/or TUDCA treatment timing in porcine oocyte during IVM. During the maturation periods, Tm and/or TUDCA as an ER stress regulators were added to the maturation medium according to the treatment timing such as IVM I (0-22 h), IVM II (22-44 h) and IVM I \& IVM II (0-44 h). were denatured at $100^{\circ} \mathrm{C}$ for 15 to $20 \mathrm{~min}$. The mixture was separated by $10 \%$ sodium dodecyl sulfate polyacrylamide gel electrophoresis (SDS-PAGE; Hoefer, Holliston, MA, USA) for $2 \mathrm{~h}$ at $20 \mathrm{~mA}$ and the resolved proteins were transferred to nitrocellulose membranes (Pall Corporation, Darmstadt, Germany) for $2 \mathrm{~h}$ at $400 \mathrm{~mA}$. The membranes were blocked using Tris-buffered saline containing $0.1 \%$ Tween 20 (TBST) supplemented with 5\% BSA for $5 \mathrm{~h}$ at room temperature. The blocked membranes were washed three times for 5 min each using TBST, and then were incubated with TBST supplemented with $2.5 \%$ BSA overnight at $4^{\circ} \mathrm{C}$ in the presence of the following primary antibodies: anti-P-eIF2 $\alpha$ (Cell Signaling Technology, Beverly, MA), anti-eIF2 $\alpha$ (Cell Signaling), anti-Bip/Grp78 (Santa Cruz Biotechnology, CA, USA), anti-ATF4 (Santa Cruz), anti- $\beta$-Actin (Santa Cruz), and anti-SESN2 (Abcam, Cambridge, MA, USA). The membranes were incubated with horseradish peroxidase (HRP)-conjugated anti-mouse and anti-goat IgG (1:5000; Santa Cruz) secondary antibodies overnight at $4^{\circ} \mathrm{C}$. Antibody binding was detected using chemiluminescence with a Bright ${ }^{\mathrm{TM}}$ ECL Kit (Advansta Inc., San Jose, CA, USA). Protein content was determined using Fusion Solo software (Vilber Lourmat, Collégien, France). Both experiments were performed at least three times.

\section{Immunofluorescence (IF) staining}

After IVM II, COCs were washed with 0.1\% PVA-PBS and fixed in $2.5 \%(\mathrm{v} / \mathrm{v})$ glutaraldehyde solution overnight at $4^{\circ} \mathrm{C}$. Then, COCs were transferred to a permeabilization solution $(0.5 \%$ Triton $\mathrm{X}-100)$ at room temperature for $1 \mathrm{~h}$. Next, COCs were washed in 0.1\% PVA-PBS three times and then blocking was performed in 0.1\% PVAPBS supplemented with $1 \% \mathrm{BSA}$ at $4^{\circ} \mathrm{C}$ for overnight. The COCs were incubated with 1:1000 diluted anti-SESN2 (10795-1-AP, Proteintech Group, Chicago, IL, USA) at $4^{\circ} \mathrm{C}$ overnight. After incubation, the COCs reacted with the secondary antibody, FITC-conjugated goat anti-rabbit IgG (Santa Cruz), diluted 1:1000 in 0.1\% PVA-PBS for 40 minutes at $37^{\circ} \mathrm{C}$ in an incubator. Immunoreactivity was observed under an $\mathrm{iRiS}^{\mathrm{TM}}$ digital cell imaging system (Logos Biosystems, Inc., Anyang, Korea).

\section{Assessment of meiotic maturation}

After IVM II (44 h), Tm and/or TUDCA treated porcine oocyte maturation was classified by nuclear stages. Oocyte were denuded by TL-HEPES medium including $0.1 \%$ hyaluronidase. Denuded oocytes were washed three times in $0.1 \%$ PVA-PBS medium and mounted on microscope slides. Oocytes were fixed for 2-3 days in acetic acid : ethanol (1:3, v/v) and were stained with $1 \%$ acetic orcein (v/v) solution for $5 \mathrm{~min}$. After $5 \mathrm{~min}$, oocytes were destained in acetic acid : glycerol : water $(1: 1: 3, \mathrm{v} / \mathrm{v} / \mathrm{v})$ solution. Then, meiotic stages were observed under a microscope (Leica, Solms, Germany).

\section{Statistical analysis}

All experiments were replicated more than three times. All values of Western blot and image experiments were presented as the mean \pm standard error of the mean (SEM). All percentage data evaluated in the present study were presented as the mean \pm standard deviation (SD). The results were analyzed using a one-way ANOVA followed by Bonferroni's Multiple Comparison Test and us- 
ing t-tests. Histogram values of densitometry analysis were obtained using the Image J software (National Institutes of Health, MD, USA). All data calculations were performed using the GraphPad Prism 5.0 software package (San Diego, CA, USA). Differences were designated significant at ${ }^{*} p<0.05,{ }^{* *} p<0.01$, and ${ }^{* * *} p<0.001$.

\section{RESULTS}

Changes in SESN2 protein level in porcine DOs, CCs, and $\mathrm{COCs}$ during IVM progression

To examine the expression of SESN2 protein in porcine DOs, CCs, and COCs after $22 \mathrm{~h}$ and $44 \mathrm{~h}$ IVM, we performed Western blot analysis. According to the result, protein level of SESN2 increased in DOs after $44 \mathrm{~h}$ IVM,
A
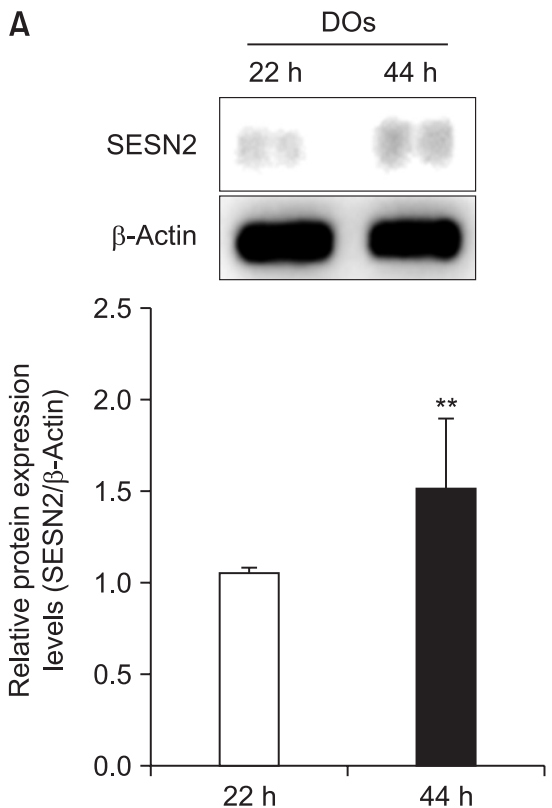

B
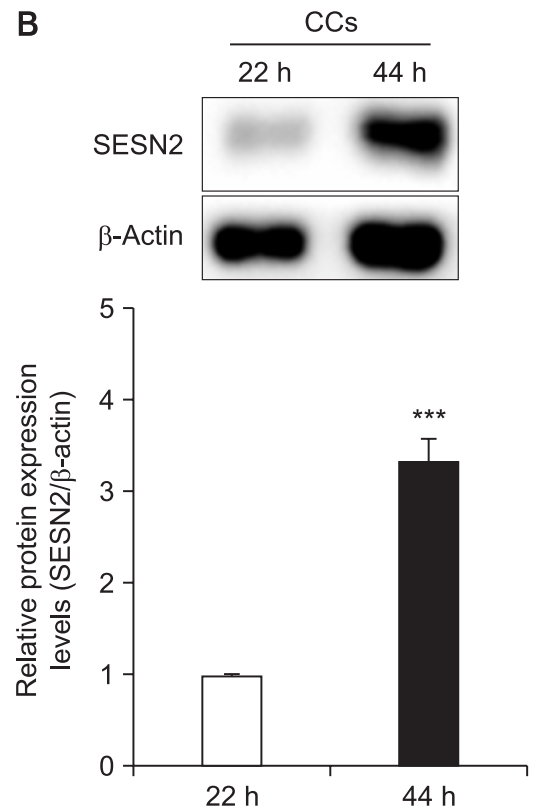

C

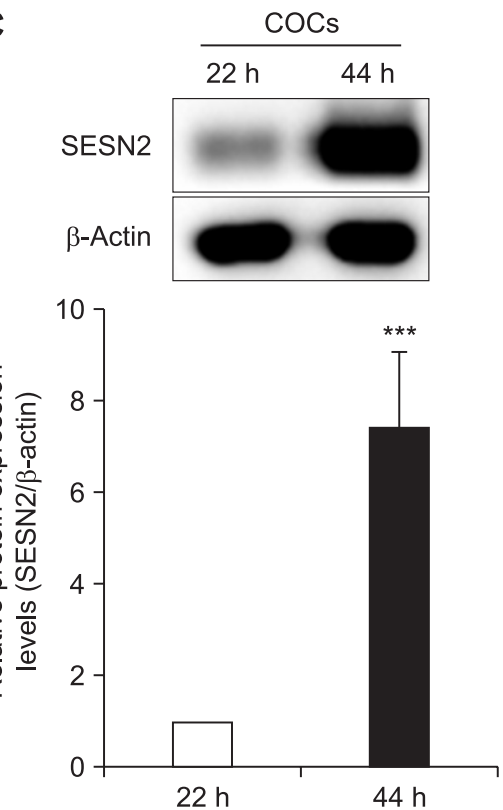

Fig. 2. Change of SESN2 protein expression in porcine DOs, CCS, and COCs during IVM, respectively. Protein level of SESN2 was detected in (A) DOs, (B) CCs, and (C) COCs during IVM by Western blot analysis. Histogram of protein level in DOs, CCs, and COCs during IVM. Relative SESN2 protein expression level was normalized to $\beta$-Actin. Data in the bar graph are means \pm SEM of independent experiments repeated at least three times. Differences were examined significant at ${ }^{* *} p<0.01,{ }^{* *} p<0.001 ; p$ values were analyzed using t-tests.
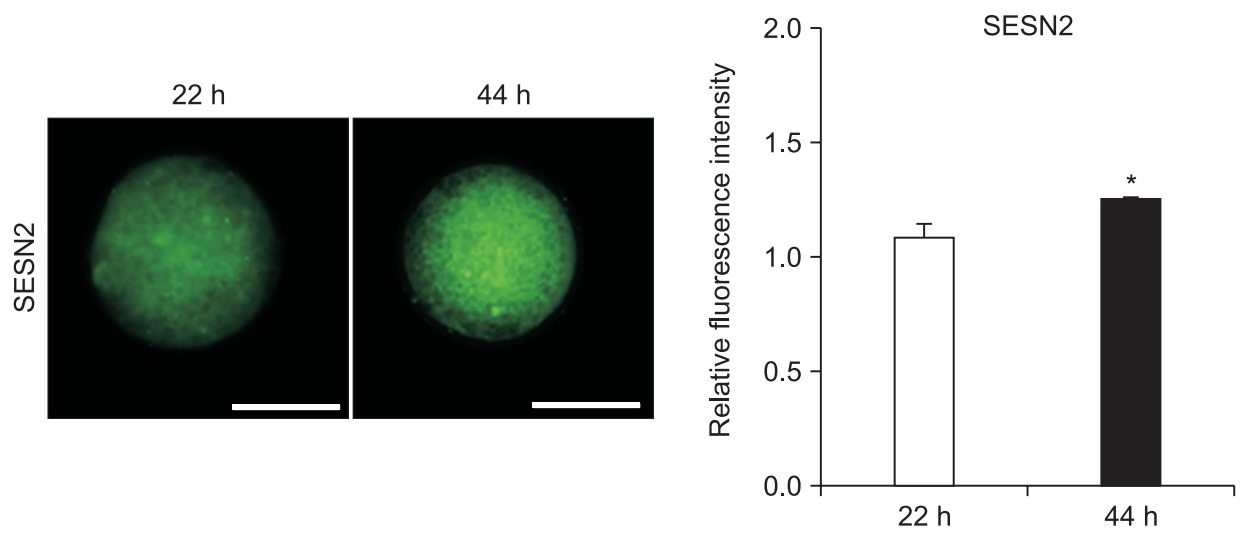

Fig. 3. Investigation of SESN2 distribution using IF staining in porcine DOs at $44 \mathrm{~h}$ after IVM. Expression pattern of stress marker protein SESN2 in porcine DOs of IVM I (22 h) and IVM II (44 h) were investigated by IF staining analysis. Histogram of fluorescence intensity level in DOs during IVM. These data in bar graph are means \pm S.D of independent experiments repeated at least three times. Differences were examined significant at ${ }^{*} p<0.05 ; p$ values were analyzed using t-tests. Scale bar is $100 \mu \mathrm{m}$. 
and matured porcine CCs and COCs were significantly increased $(p<0.05)$ compared to $22 \mathrm{~h}$ after IVM group (Fig. $2 \mathrm{~A}-2 \mathrm{C}$ ). Then, to observe distribution of SESN2 protein expression in DOs after $44 \mathrm{~h}$ of IVM, we performed IF staining analysis. As shown in Fig. 3, green fluorescence intensity of SESN2 in cytoplasm of porcine oocyte at 44 $\mathrm{h}$ was increased compared with oocyte of $22 \mathrm{~h}$ group. In addition, the SESN2 fluorescence expression was observed to be equally distributed in the cytoplasm of porcine oocyte. These results demonstrated that SESN2 is expressed

Table 1. Meiotic maturation according to the $\mathrm{Tm} 1$ and $5 \mu \mathrm{g} / \mathrm{mL}$ treatment in porcine oocyte

\begin{tabular}{cccccc}
\hline \multirow{2}{*}{$\begin{array}{c}\text { Tm } \\
(\mu \mathrm{g} / \mathrm{mL})\end{array}$} & $\begin{array}{c}\text { No. of oocytes } \\
\text { examined }\end{array}$ & \multicolumn{4}{c}{ \% of oocytes (n) } \\
\cline { 3 - 6 } & 144 & GV & GVBD & M I & M II \\
\hline Con & 152 & $1.4 \pm 1.6(3)$ & $5.9 \pm 4.2(8)$ & $19.4 \pm 1.9(28)$ & $72.5 \pm 4.2(105)^{a}$ \\
1 & 147 & $5.2 \pm 2.1(8)$ & $10.0 \pm 1.8(15)$ & $25.5 \pm 2.7(39)$ & $59.3 \pm 2.4(90)^{b}$ \\
5 & $10.9 \pm 2.0(16)$ & $18.4 \pm 3.1(27)$ & $24.6 \pm 2.8(36)$ & $46.2 \pm 4.1(68)^{c}$ \\
\hline
\end{tabular}

Data are the means \pm standard deviation (SD).

a,b, $V$ Values with different superscripts denote a significant difference $(p<0.05)$.
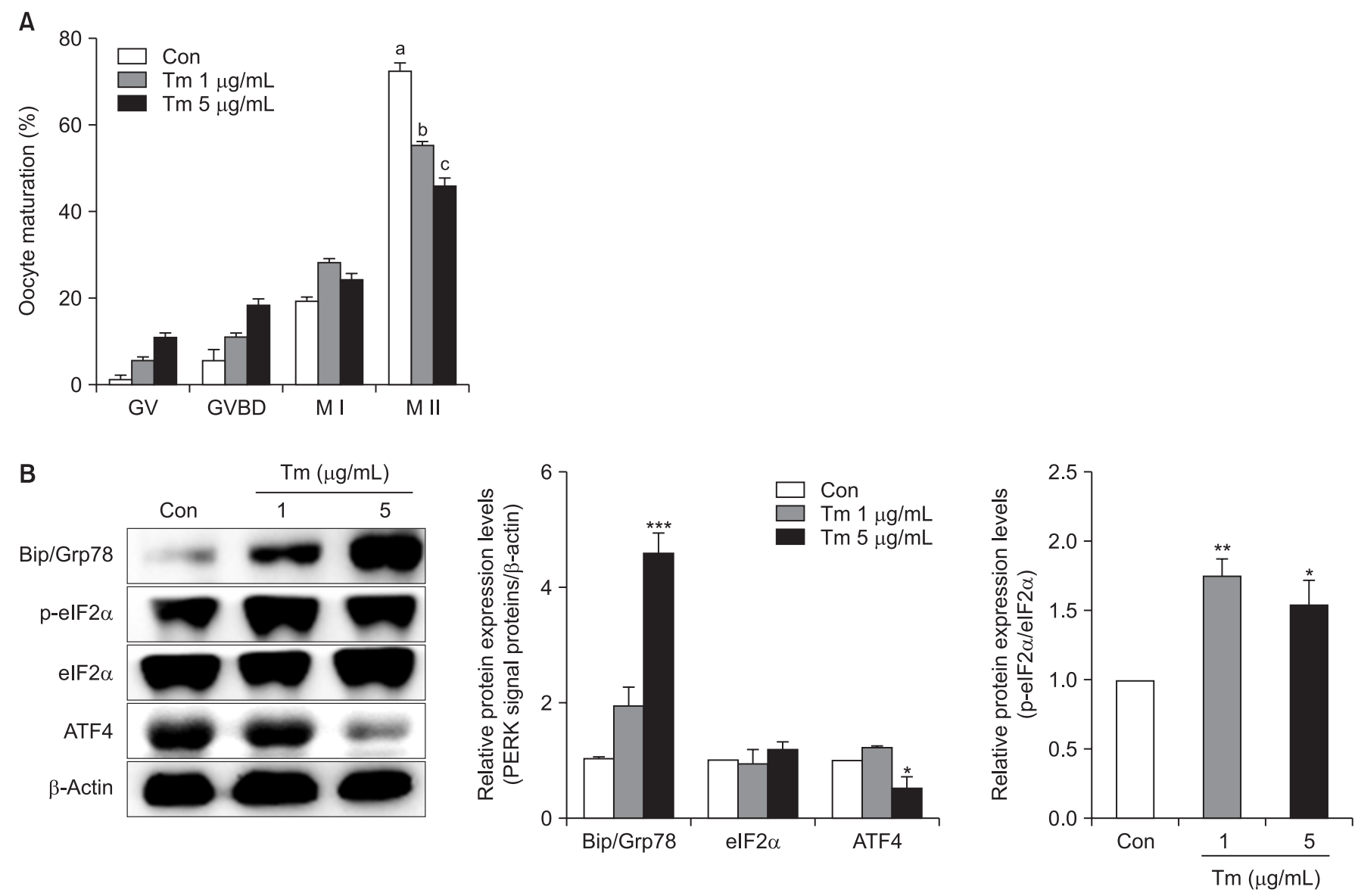

Fig. 4. Expressions of PERK signaling and meiotic maturation in Tm treated porcine COCs. (A) Table 1 and histogram of meiotic maturation depending on the treated with various $\mathrm{Tm}$ concentration (1 and $5 \mu \mathrm{g} / \mathrm{mL}$ ). These data in bar graph are means \pm SD of independent experiments repeated at least three times. ${ }^{a, b, c}$ Values with different superscripts denote a significant difference $(p<0.05)$. (B) Expressions of PERK signaling (Bip/Grp78, p-elF2 $\alpha$, elF2 $\alpha$ and ATF4) were detected in matured COCs by Western blot analysis. Histogram of protein levels in COCs. Relative of these protein expressions were normalized to $\beta$-Actin. These data in bar graph are means \pm SEM of independent experiments repeated at least three times. Differences were examined significant at ${ }^{*} p<0.05,{ }^{* *} p<0.01,{ }^{* * *} p$ $<0.001 ; p$ values were analyzed using Bonferroni's Multiple Comparison Test. 
in DOs, CCs, and COCs respectively. Then, SESN2 is localized in the entire cytoplasm of porcine oocyte. Based on these results, we determined that the matured COCs after $44 \mathrm{~h}$ of IVM in pigs can be used for the subsequent experiments as sample type.

\section{Effects of inducing ER stress by Tm during meiotic maturation of porcine oocyte and PERK signaling activation in porcine COCs}

In our previous study, we demonstrated that ER stress affects porcine oocyte maturation during the $44 \mathrm{~h}$ after IVM. In addition, ER stress-induced UPR signaling pathway activation occurs in $\mathrm{Tm} 1 \mu \mathrm{g} / \mathrm{mL}$ treated porcine COCs during IVM (Park et al., 2018). According to our previous results, we investigated whether meiotic maturation is reduced by Tm treatment concentration ( 1 and 5 $\mu \mathrm{g} / \mathrm{mL}$ ) in porcine oocyte. As expected, as shown in Table 1 and Fig. 4A, meiotic maturation rate significantly decreased $(p<0.05)$ in Tm treatment concentration dependent manner compared with control group at $44 \mathrm{~h}$ of IVM (Control; $72.5 \pm 4.2 \%$ vs Tm $1 \mu \mathrm{g} / \mathrm{mL} ; 59.3 \pm 2.4 \%$, Tm 5 $\mu \mathrm{g} / \mathrm{mL} ; 46.2 \pm 4.1 \%)$. Under increase in ER stress by $\mathrm{Tm}$ treatment, the protein levels of Bip/Grp78, p-eIF2 $\alpha$ and ATF4 were increased in matured porcine COCs (Fig. 4B).

\section{Effects of TUDCA treatment on meiotic maturation of porcine COCs}

We investigated the effects of TUDCA as an ER stress inhibitor on meiotic maturation in porcine oocyte. Matured porcine oocytes in IVM medium are supplemented in various concentrations $(50,100$ and $200 \mu \mathrm{M})$ of TUDCA for 44 h. As shown in Table 2 and Fig. 5A, meiotic maturation also significantly increased $(p<0.05)$ in TUDCA 200 $\mu \mathrm{M}$ treated group compared with other groups at $44 \mathrm{~h}$ of IVM (Control; $70.6 \pm 5.8 \%$, TUDCA $50 \mu \mathrm{M} ; 76.3 \pm 2.8 \%$, $100 \mu \mathrm{M} ; 79.8 \pm 6.8 \%, 200 \mu \mathrm{M} ; 81.3 \pm 5.3 \%)$. These results showed that the reducing ER stress by TUDCA treatment improved the meiotic maturation of porcine COCs after $44 \mathrm{~h}$ of IVM.

\section{Investigation of SESN2 protein expression in porcine COCs with Tm and/or TUDCA treatment during IVM}

Based on the results, to confirm the recovery effects of TUDCA response to $\mathrm{Tm}$, we measured the meiotic maturation rate in porcine oocyte after TUDCA and/or Tm treatment. As expected, meiotic maturation rate were significantly recovered $(p<0.05)$ in TUDCA treatment after Tm pre-treated group (Tm + TUDCA) compared with only Tm treated groups (Control; $72.6 \pm 2.8 \%$, only Tm $1 \mu \mathrm{g} /$ $\mathrm{mL} ; 60.2 \pm 1.7 \%$, only TUDCA $200 \mu \mathrm{M} ; 78.8 \pm 2.9 \%$, Tm + TUDCA; $70.3 \pm 3.8 \%$ ) (Table 3 and Fig. 5B). Interestingly, protein levels of SESN2 showed the same pattern as that of meiotic maturation rate in $44 \mathrm{~h}$ after IVM (Fig. 5C). And then, SESN2 protein expression were significantly decreased in only TUDCA treated COCs. These results suggested that TUDCA treatment recovered the reducing meiotic maturation after Tm-induced ER stress in maturing COCs.

\section{DISCUSSION}

In present study, we investigated the protein level of SESN2 in denuded oocyte (DOs), cumulus cells (CCs), and cumulus-oocyte complex (COCs) during in vitro maturation (IVM) in porcine oocytes. Interestingly, SESN2 protein levels were significantly increased in porcine DOs, CCs and COCs according to the IVM progression. In addition, our previous study demonstrated that the ER stress and UPR signal factors expression were increased in matured porcine COCs. As shown in the Fig. 3, under Tm-induced ER stress, PERK signal pathways activated and meiotic maturation reduced in porcine COCS. On the contrary,

Table 2. Effects of reducing ER stress by TUDCA treatment on meiotic maturation of porcine oocyte in $44 \mathrm{~h}$ after IVM

\begin{tabular}{cccccc}
\hline \multirow{2}{*}{$\begin{array}{c}\text { TUDCA } \\
(\mu \mathrm{M})\end{array}$} & $\begin{array}{c}\text { No. of oocytes } \\
\text { examined }\end{array}$ & GV & \multicolumn{3}{c}{ \% of oocytes $(\mathrm{n})$} \\
\cline { 3 - 6 } & 161 & $2.6 \pm 2.2(5)$ & $10.1 \pm 2.8(16)$ & $16.2 \pm 2.4(26)$ & $70.6 \pm 5.8(114)^{\mathrm{a}}$ \\
\hline Con & 151 & $4.0 \pm 1.4(6)$ & $5.2 \pm 1.8(8)$ & $14.6 \pm 2.8(22)$ & $76.3 \pm 2.8(115)^{\mathrm{a}}$ \\
50 & 178 & $5.2 \pm 1.5(9)$ & $4.4 \pm 1.5(8)$ & $10.7 \pm 4.7(19)$ & $79.8 \pm 6.8(142)^{\mathrm{a}}$ \\
100 & 147 & $4.3 \pm 2.1(6)$ & $5.4 \pm 3.4(8)$ & $9.1 \pm 2.4(13)$ & $81.3 \pm 5.3(120)^{b}$ \\
\hline
\end{tabular}

Data are the means \pm SD.

a,b Values with different superscripts denote a significant difference $(p<0.05)$. 
A

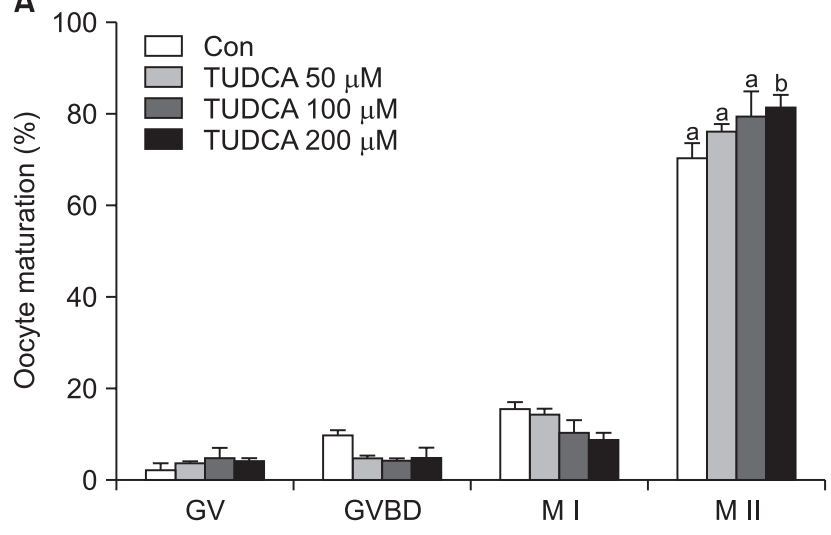

C

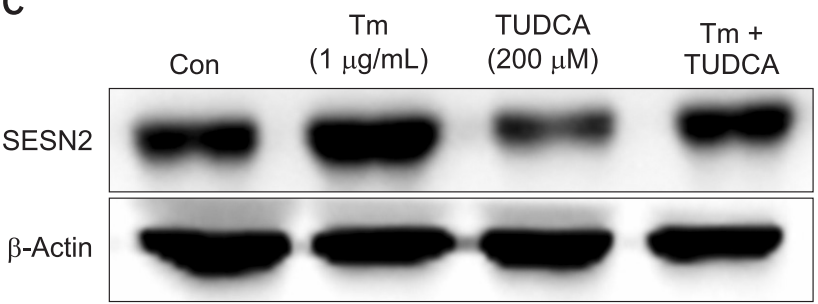

B
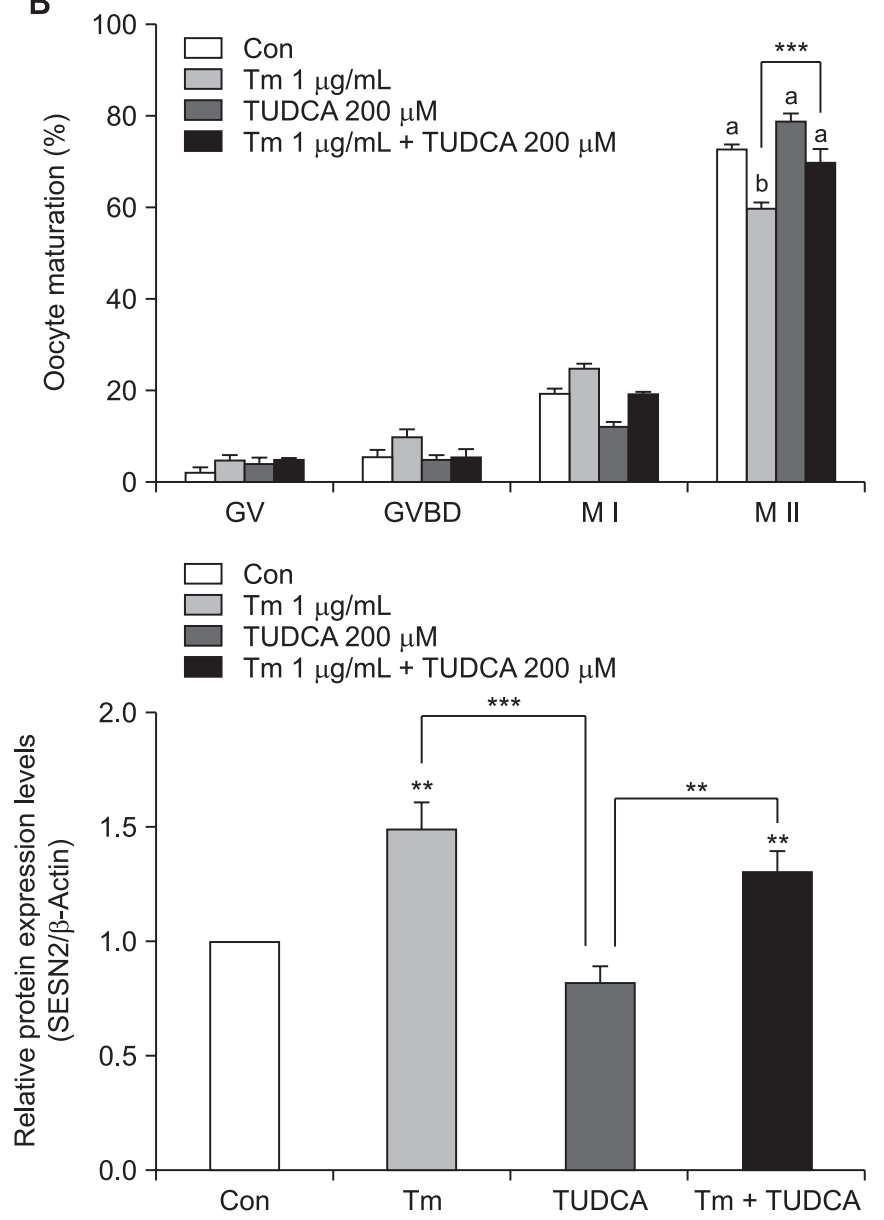

Fig. 5. Protein levels of SESN2 and meiotic maturation in TUDCA and/or Tm treated porcine COCs. (A) Table 2 and histogram of meiotic maturation depending on the treated with various TUDCA concentration (50, 100 and $200 \mu \mathrm{M})$. ${ }^{\text {abb }}$ Values with different superscripts denote a significant difference $(p<0.05)$. (B) Table 3 and histogram of meiotic maturation depending on the treated with Tm and/or TUDCA concentration (only Tm $1 \mu \mathrm{g} / \mathrm{mL}$, only TUDCA $200 \mu \mathrm{M}$ and Tm + TUDCA; TUDCA treatment after Tm pre-treated). These data in bar graph are means \pm SD of independent experiments repeated at least three times. ${ }^{a, b} V a l u e s$ with different superscripts denote a significant difference $(p<0.05)$. (C) Expressions of SESN2 protein were detected in matured COCs after Tm and/or TUDCA treatment by Western blot analysis. Histogram of protein levels in COCs. Relative of these protein expressions were normalized to $\beta$-Actin. These data in bar graph are means \pm SEM of independent experiments repeated at least three times. Differences were examined significant at ${ }^{* *} p<0.01,{ }^{* *} p<0.001 ; p$ values were analyzed using Bonferroni's Multiple Comparison Test.

Table 3. Changes in meiotic maturation rate in Tm and/or TUDCA treated porcine oocyte

\begin{tabular}{ccccccc}
\hline \multirow{2}{*}{$\begin{array}{c}\text { Tm } \\
(1 \mu \mathrm{g} / \mathrm{mL})\end{array}$} & \multirow{2}{*}{$\begin{array}{c}\text { TUDCA } \\
(\mathbf{2 0 0} \mu \mathrm{M})\end{array}$} & $\begin{array}{c}\text { No. of oocytes } \\
\text { examined }\end{array}$ & & \multicolumn{3}{c}{ \% of oocytes $(\mathrm{n})$} \\
\cline { 4 - 7 } & - & 158 & $2.0 \pm 2.7(4)$ & $5.3 \pm 3.7(8)$ & $19.4 \pm 2.8(31)$ & $72.6 \pm 2.8(115)^{\mathrm{a}}$ \\
+ & - & 153 & $5.1 \pm 1.7(8)$ & $10.0 \pm 2.3(15)$ & $24.6 \pm 2.5(38)$ & $60.2 \pm 1.7(92)^{\mathrm{b}}$ \\
- & + & 130 & $4.1 \pm 2.0(5)$ & $5.1 \pm 1.8(7)$ & $12.0 \pm 2.6(16)$ & $78.8 \pm 2.9(102)^{\mathrm{a}}$ \\
+ & + & 150 & $4.8 \pm 1.7(7)$ & $5.8 \pm 3.4(9)$ & $19.2 \pm 2.0(29)$ & $70.3 \pm 3.8(105)^{\mathrm{a}}$ \\
\hline
\end{tabular}

Data are the means \pm SD.

a,b Values with different superscripts denote a significant difference $(p<0.05)$.

ER stress inhibitor, TUDCA treated COCs showed improvement of meiotic maturation in porcine oocyte after $44 \mathrm{~h}$ of IVM. Based on these results, we investigated the
SESN2 protein levels, which affected the according to the ER stress by ER stress regulator during oocyte maturation in pigs. These observations provide the first evidence re- 


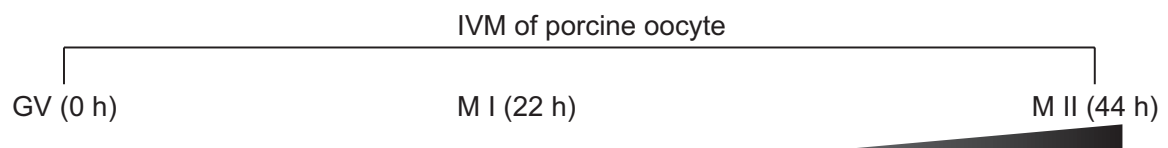

ER-stress $\uparrow$

UPR signaling pathways activation $\uparrow$
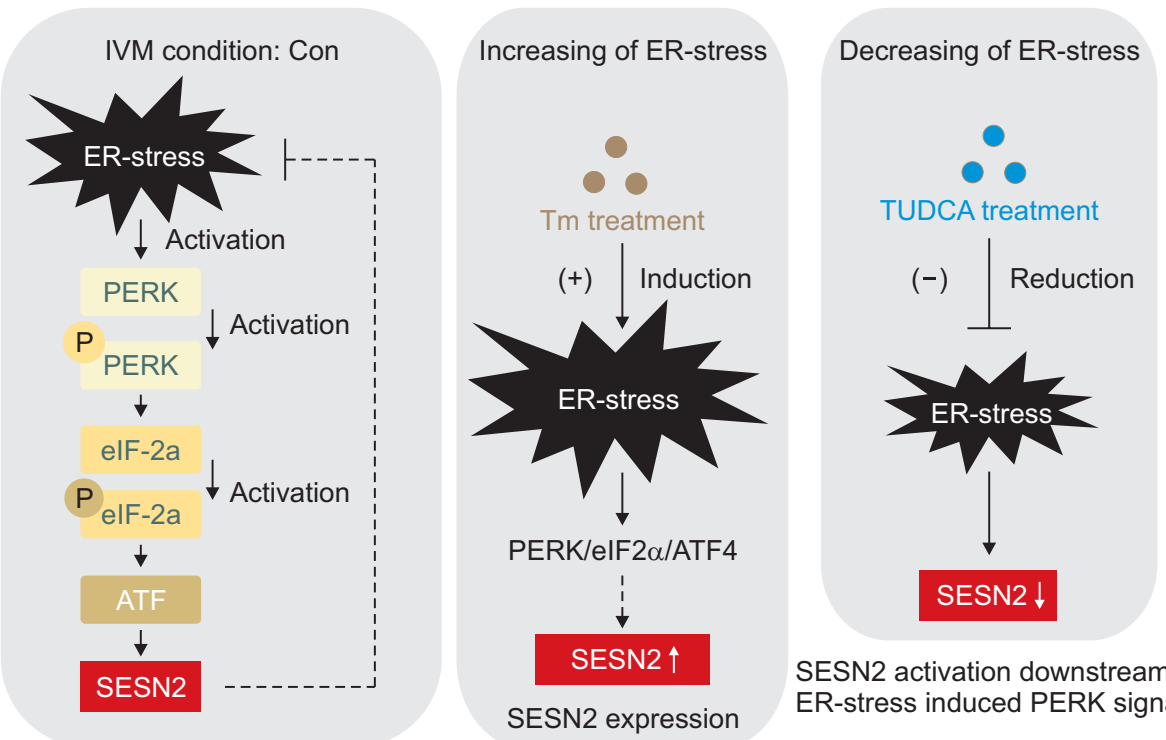

SESN2 activation downstream of ER-stress induced PERK signaling
Fig. 6. Graphical summary. During porcine oocyte IVM, UPR signaling activation response to ER stress occurs in pigs. When increased in ER stress by $\mathrm{Tm}$, protein levels of SESN2 as a stress proteins were significantly increased in matured COCs after $44 \mathrm{~h}$ of IVM. But, contrary to $\mathrm{Tm}$ treated condition, only TUDCA as an ER stress inhibitor treated COCs showed the reducing SESN2 protein expression in porcine COCs. Interestingly, increased SESN2 protein levels by $\mathrm{Tm}$ were recovered in TUDCA treated COCs. Based on these results, the SESN2 expression as a stress mediator response to ER stress is related to the activity of PERK signaling pathways in porcine oocyte maturation. garding the basic mechanisms connecting SESN2 protein expression and PERK signaling activation response to ER stress in porcine COCs and oocyte maturation in vitro.

The activity of UPR signaling pathways plays an important role in oocyte maturation (Zhang et al., 2012), and embryonic development (Basar et al., 2014), which is related to chaperone functions, progesterone production and cellular apoptosis in pigs. To prevent ER stress damage, PERK signaling induces anti-oxidative genes expressions (Marciniak et al., 2004). These roles of antioxidant for reduction of ROS is important in porcine oocyte maturation and cumulus cells expansion (Yang et al., 2019). Additionally, PERK signaling is critical in reducing ER stress by attenuation of translation (Hetz and Papa, 2018). PERK signal activation plays important role in oocyte maturation, in vitro fertilization (IVF) process, and blastocyst development (Latham KE., 2016). The expressions of PERK-related proteins were increased during IVM progression in CCs and COCs (data not shown). In addition, PERK signaling was activated by Tm-induced ER stress in porcine COCs. Previous study has reported the reduction of blastocysts formation in PERK knockout mouse (Hard- ing et al., 2001). Based on these results, we confirmed that the activation of PERK signaling is a marker for protecting ER stress damage in porcine oocyte during IVM.

SESN2 as a stress mediator is known to increase expression in response to various stresses including oxidative stress and ER-stress (Wang et al., 2017). However, the expression pattern of SESN2 on oocytes maturation in pigs is not yet clearly understood. In addition, expression of SESN2 protein was increased in CCs and COCs during IVM progression and its localization to the cytoplasm in porcine DOs was confirmed using immunofluorescence (IF) staining. Increase of SESN2 expression and PERK signaling activation improved in matured porcine COCs after $44 \mathrm{~h}$ of IVM. Based on these results, we speculated that SENS2 protein is expressed as a downstream of PERK signaling pathways in porcine oocytes. To confirm the relationship between ER-stress and SESN2 in porcine oocytes, we measured the meiotic maturation and expression of PERK-related protein using Tm as ER-stress inducer. Under severe ER stress, UPR becomes cytotoxic, including apoptosis rather than being protective (Zeeshan et al., 2016). As shown in Fig. 4, ATF4 expression of PERK 
signaling proteins in $\mathrm{Tm} 5 \mu \mathrm{g} / \mathrm{mL}$ treated group decreased compared with $\mathrm{Tm} 1 \mu \mathrm{g} / \mathrm{mL}$ treated group.

We confirmed that meiotic maturation was significantly increased in $200 \mu \mathrm{M}$ TUDCA treated COCs. In addition, we confirmed the changes in meiotic maturation and expression of SESN2 protein according to Tm and/or TUDCA treatment in porcine COCs. Previous study reported that the TUDCA treatment effectively suppressed SESN2 expressions in ER stress induced mammalian hepatocyte (Ding et al., 2016). Interestingly, reduced SESN2 protein levels by Tm were recovered in TUDCA treated COCs. In previous study, SESN2-deficient cells were induced to be apoptotic by ER stress mediated apoptosis (Park et al., 2014). Based in these results, we speculated the correlation between SESN2 protein expression and PERK signaling activation response to ER stress in matured porcine oocyte during IVM.

In conclusion, we confirmed that inducing Tm reduced PERK signaling markers and SESN2 protein expression in matured COCs of pigs (Fig. 6). In addition, reducing ER stress condition by TUDCA, porcine COCs showed the improvement of meiotic maturation and reduction of SESN2 protein level in $44 \mathrm{~h}$ of IVM. Therefore, we speculated that SESN2 is related to ER stress in meiotic maturation by a stress regulator through the PERK signaling pathway in pigs. Moreover, we suggested that SESN2 protein might affect PERK signaling activity response to ER stress on porcine oocyte maturation.

\section{CONFLICTS OF INTEREST}

No potential conflict of interest relevant to this article was reported.

\section{ACKNOWLEDGEMENTS}

This work was supported by grants from the Basic Science Research Program (NRF-2018R1C1B002922 and NRF-2018R1D1A1B07044173), Cooperative Research Program for Agriculture Science and Technology Development (PJ01269503), and the Agri-Bio industry Technology Development Program (316037-04) through the Ministry of Education, the Rural Development Administration, and the Ministry of Agriculture, Food and Rural Affairs, Republic of Korea.

\section{ORCID}

Hyo-Jin Park: https://orcid.org/0000-0003-3287-2914

In-Su Kim: https://orcid.org/0000-0001-5703-092X

Jin-Woo Kim: https://orcid.org/0000-0002-0089-680X

Seul-Gi Yang: https://orcid.org/0000-0003-3247-2443

Min-Ji Kim: https://orcid.org/0000-0002-3443-4865

Deog-Bon Koo: https://orcid.org/0000-0001-7825-9598

\section{REFERENCES}

Basar M, Bozkurt I, Guzeloglu-Kayisli O, Sozen B, Tekmen I, Schatz F, Arici A, Lockwood CJ and Kayisli UA. 2014. Unfolded protein response prevents blastocyst formation during preimplantation embryo development in vitro. Fertil Steril. 102(6):1777-1784.

Bravo R, Parra V, Gatica D, Rodriguez AE, Torrealba N, Paredes $F$ and Quest AF. 2013. Endoplasmic reticulum and the unfolded protein response: dynamics and metabolic integration. Int Rev Cell Mol Biol. 301:215-290.

Brewer JW and Diehl JA. 2000. PERK mediates cell-cycle exit during the mammalian unfolded protein response. Proc Nati Acad Sci U S A. 97(23):12625-12630.

Ding B, Parmigiani A, Divakaruni AS, Archer K, Murphy AN and Budanov AV. 2016. Sestrin2 is induced by glucose starvation via the unfolded protein response and protects cells from non-canonical necroptotic cell death. Sci Rep. 6:22538.

Harding HP, Zeng H, Zhang Y, Jungries R, Chung P, Plesken H, Sabatini DD and Ron D. 2001. Diabetes mellitus and exocrine pancreatic dysfunction in perk-/- mice reveals a role for translational control in secretory cell survival. Mol Cell. 7(6):1153-1163.

Jin HR, Du CH, Wang CZ, Yuan CS and Du W. 2019. Ginseng metabolite Protopanaxadiol induces Sestrin2 expression and AMPK activation through GCN2 and PERK. Cell Death Dis. 10(4):311.

Kaufman RJ. 1999. Stress signaling from the lumen of the endoplasmic reticulum: coordination of gene transcriptional and translational controls. Genes Dev. 13(10):1211-1233.

Kim GT, Lee SH, Kim JI and Kim YM. 2014. Quercetin regulates the sestrin 2-AMPK-p38 MAPK signaling pathway and induces apoptosis by increasing the generation of intracellular ROS in a p53-independent manner. Int J Mol Med. 33(4): 863-869.

Kim H, An S, Ro SH, Teixeira F, Park GJ, Kim C, Cho CS, Kim JS, Jakob U, Lee JH and Cho US. 2015. Janus-faced Sestrin2 controls ROS and mTOR signalling through two separate functional domains. Nat Commun. 27(6):10025.

Kim JS, Song BS, Lee KS, Kim DH, Kim SU, Choo YK, Chang KT and Koo DB. 2012. Tauroursodeoxycholic acid enhances the pre-implantation embryo development by reducing apoptosis in pigs. Reprod Domest Anim. 47(5):791-798.

Kitagawa Y, Suzuki K, Yoneda A and Watanabe T. 2004. Effects 
of oxygen concentration and antioxidants on the in vitro developmental ability, production of reactive oxygen species (ROS), and DNA fragmentation in porcine embryos. Theriogenology. 62(7):1186-1197.

Latham KE. 2016. Stress signaling in mammalian oocytes and embryos: a basis for intervention and improvement of outcomes. Cell Tissue Res. 363(1):159-167.

Lin T, Diao YF, Choi HS, Oqani RK, Kang JW, Lee JE and Jin DI. 2015. Procedure used for denuding pig oocytes influences oocyte damage, and development of in vitro and nuclear transfer embryos. Anim Reprod Sci. 152:65-76.

Lin T, Lee JE, Kang JW, Shin HY, Lee JB and Jin DI. 2019. Endoplasmic Reticulum (ER) Stress and Unfolded Protein Response (UPR) in Mammalian Oocyte Maturation and Preimplantation Embryo Development. Int J Mol Sci. 20(2):409.

Luo S, Mao C, Lee B and Lee AS. 2006. GRP78/BiP is required for cell proliferation and protecting the inner cell mass from apoptosis during early mouse embryonic development. Mol Cell Biol. 26(15):5688-5697.

Marciniak SJ, Yun CY, Oyadomari S, Novoa I, Zhang Y, Jungreis R, Nagata K, Harding HP and Ron D. 2004. CHOP induces death by promoting protein synthesis and oxidation in the stressed endoplasmic reticulum. Genes Dev. 18(24):30663077.

Park HJ, Park JY, Kim JW, Yang SG, Jung JM, Kim MJ, Kang MJ, Cho YH, Wee G, Yang HY, Song BS, Kim SU and Koo DB. 2018. Melatonin improves the meiotic maturation of porcine oocytes by reducing endoplasmic reticulum stress during in vitro maturation. J. Pineal Res. 64(2):E12458.

Park HJ, Park SY, Kim JW, Yang SG, Kim MJ, Kang MJ, Jegal HG, Kim IS, Choo YK and Koo DB. 2018. Melatonin Improves Oocyte Maturation and Mitochondrial Functions by Reducing Bisphenol A-Derived Superoxide in Porcine Oocytes In Vitro. Int J Mol Sci. 19(11):3422.

Park HW, Park H, Ro SH, Jang I, Semple IA, Kim DN, Kim M, Nam M, Zhang D, Yin L and Lee JH. 2014. Hepatoprotective role of Sestrin2 against chronic ER stress. Nat Commun. 5: 4233.

Rasheva VI and Domingos PM. 2009. Cellular responses to endoplasmic reticulum stress and apoptosis. Apoptosis. 14(8): 996-1007.

Rodrigues CM, Linehan-Stieers C, Keene CD, Ma X, Kren BT,
Low WC and Steer CJ. 2000. Tauroursodeoxycholic acid partially prevents apoptosis induced by 3-nitropropionic acid. J Neurochem. 75(6):2368-2379.

Schröder M. 2008. Endoplasmic reticulum stress responses. Cell Mol Life Sci. 65(6):862-894.

Shin BY, Jin SH, Cho IJ and Ki SH. 2012. Nrf2-ARE pathway regulates induction of Sestrin-2 expression. Free Radic Biol Med. 53(4):834-841.

Walter P and Ron D. 2011. The unfolded protein response: from stress pathway to homeostatic regulation. Science. 334(6059):1081-1086.

Wang M, Xu Y, Liu J, Ye J, Yuan W, Jiang H, Wang Z, Jiang H and Wan J. 2017. Recent insights into the biological functions of sestrins in health and disease. Cell Physiol Biochem. 43(5): 1731-1741.

Yang SG, Park HJ, Lee SM, Kim JW, Kim MJ, Kim IS, Jegal HG and Koo DB. 2019. Reduction of mitochondrial derived superoxide by Mito-TEMPO improves porcine oocyte maturation in vitro. Journal of Animal Reproduction \& Biotechnology. 34(1):10-19.

Yang Y, Pei X, Jin Y, Wang Yand Zhang C. 2016. The roles of endoplasmic reticulum stress response in female mammalian reproduction. Cell Tissue Res. 363(3):589-597.

Ye J, Palm W, Peng M, King B, Lindsten T, Li MO, Koumenis C and Thompson CB. 2015. GCN2 sustains mTORC1 suppression upon amino acid deprivation by inducing Sestrin2. Genes Dev. 29(22):2331-2336.

Zeeshan H, Lee G, Kim HR and Chae HJ. 2016. Endoplasmic reticulum stress and associated ROS. Int J Mol Sci. 17(3):327.

Zhang J, Morris Jr MW, Dorsett-Martin WA, Drake LC and Anderson CD. 2013. Autophagy is involved in endoplasmic reticulum stress-induced cell death of rat hepatocytes. J Surg Res. 183(2):929-935.

Zhang JY, Diao YF, Oqani RK, Han RX and Jin DI. 2012. Effect of endoplasmic reticulum stress on porcine oocyte maturation and parthenogenetic embryonic development in vitro. Biol Reprod. 86(4):128.

Zhao N, Liu XJ, Li JT, Zhang L, Fu Y, Zhang YJ, Chen RX, Wei XQ, Wang R and Wang Y. 2015. Endoplasmic reticulum stress inhibition is a valid therapeutic strategy in vitrifying oocytes. Cryobiology. 70(1):48-52. 